\title{
Evaluation of the Mechanical Resistance of a Powder-powder Coating by Modulated Dry Feed Particle Size Analysis
}

\author{
Ana Vilela, Lucia Concepcion, Philippe Accart, Alain Chamayou, Michel Baron, John A. Dodds*
}

\begin{abstract}
Dry powder coating is a powerful method for modifying the surface properties of particles whilst retaining their essential bulk properties. The coating of a host powder by guest particles can be achieved via different means ranging from simple stirring together of the two components, to high energy impact coating in devices such as a Nara Hybridizer or Turbula Mixer. The quality of the powder coating resulting from such methods is an important parameter, which depends both on the force of the mechanical action used, and also the physico-chemical interactions between the host and guest particles. Here, a method for evaluating the strength of host-guest interactions based on particle size analysis using a standard Malvern Mastersizer with dry powder feed is presented. Basically, the liberation of small guest particles
\end{abstract}

from host particles can be achieved by increasing the air pressure, and the presence of liberated fine particles mixed with guest particles can be detected from differences in volume and number distributions. Complementary examination is undertaken using an environmental scanning electron microscope (ESEM). This technique has been used to evaluate the interaction strength of a coating of hydrophobic magnesium stearate on a herbal extract (Harpagophytum). It is shown that effective coating can be achieved by three different means: simple stirring, a Turbula Mixer and by using a Nara Hybridizer. The respective strength of the coatings is greater with increasing mechanical force used for the process. The method is proposed as a simple means of evaluating powder-powder coating processes.

Keywords: dry particle coating, harpagophytum, interaction strength, magnesium stearate

\section{Introduction}

The creation of highly functional materials by combining different powders can be of great scientific importance. Surface modification of powders is a recognized method because it allows the control and improvement of powder properties such as: wettability, flowability, dispersibility, color, taste, flavor, electrostatic, optical and rheological properties, etc. This provides a wide

A. Vilela, L. Concepcion, P. Accart, A. Chamayou, M. Baron, J. A. Dodds,

Ecole des Mines d'Albi-Carmaux, Chemical Engineering Laboratory for Particulate Solids UMR CNRS 2392, 81013 Albi cedex 09 (France).

E-mail: Vilela@enstimac.fr field for research and applications, e.g., cosmetics, pharmaceuticals, ceramics, foods, cements, inks, metallurgy, etc. [1]. Typically, coating processes are undertaken by wet methods such as fluidized bed coaters or pan granulation coaters. However dry phase processing has several advantages compared with the wet methods and there are now different technologies available for covering core particles with fine particles. Although possessing different methods of applying mechanical forces, each of these machines allow the de-agglomeration of the guest particles and their adhesion on the surface of the host particle. The type of coating (continuous or discrete) depends on the properties and size of the host and guest particles as well as the operating conditions of the device used [2]. Normally a good coating requires a particle size ratio of greater than 10 between the coating powder and the host particles, thus making it useful for preparing ordered mixtures when close control of low 
concentration powder dosing is required. A complete review explaining the main types of dry coating devices is presented in [3].

In this paper, fine particles of magnesium stearate (MgSt) were used to coat coarse harpagophytum extract particles by high speed rotational impact blending in a hybridizer. The strength of the interaction between host and guest particles was determined by the variation of number size distributions measured using a Malvern Mastersizer, with variation of the dispersion pressure of the dry powder feed. The results obtained were compared with the ordinary mixture prepared by both manual shaking and with a Turbula Mixer.

\section{Materials and Methods}

\subsection{Materials}

Harpagophytum particles (volume mean diameter $75 \mu \mathrm{m}$, mp $120-150^{\circ} \mathrm{C}$ ) used as the core component were coated with magnesium stearate (volume mean diameter $2 \mu \mathrm{m}, \mathrm{mp} 130-160^{\circ} \mathrm{C}$ ). Harpagophytum is a plant originating from Southern Africa, especially from the Kalahari Desert and the steppes of Namibia. The extract of Harpagophytum is widely used in Europe to treat inflammation [4]. Magnesium stearate is a fine, white, greasy powder widely used in pharmaceutical formulations as a lubricant.

The quantity of guest particles $(7 \%(\mathrm{w} / \mathrm{w}))$ is calculated to correspond to the case of hexagonal close packing on the host surface [5]. In pharmaceutical applications, the normal quantity of magnesium stearate used is $1 \%$, and consequently this concentration was chosen for the current study.

\subsection{Methods}

A hybridizer (type NHS-0, Nara Machinery) as schematically shown in Figure 1 was used to prepare coated particles. The processing can be summarized as follows: particles inside the chamber of the hybridizer are mixed and circulated in a high speed air stream caused by the rotor, and are hit repeatedly with other particles, the blades of the rotor and the wall of the stator. As a result of this mechanical action, small particles become fixed on the surface of the larger particles. The chamber is surrounded with a jacket for circulating cooling or heating fluid. The operating variables of the hybridizer are the rotational speed (up to $16000 \mathrm{rpm}$ ) and the treatment time (30 s to $10 \mathrm{~min}$ ) [2]. In the experiments reported here, the rotational speed used was $4800 \mathrm{rpm}$ for a treatment time of $4 \mathrm{~min}$.

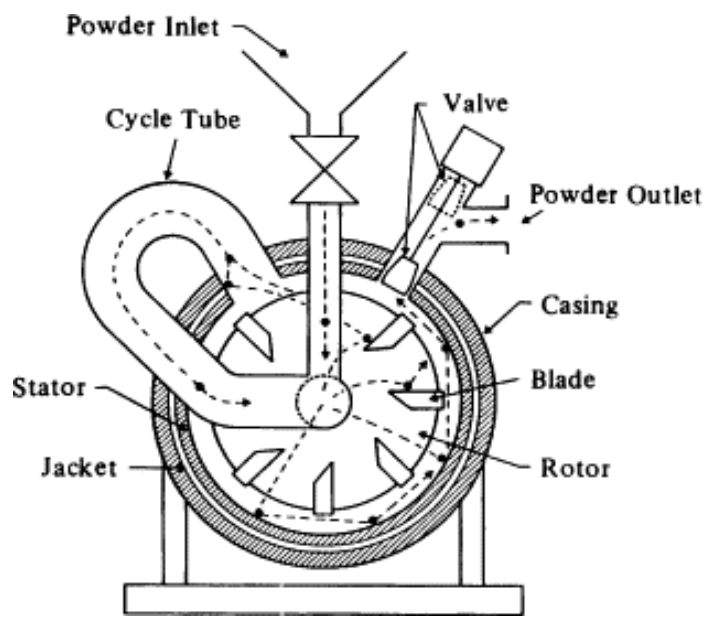

Fig. 1: Schematic diagram of the Hybridizer [7].

Coating was also performed by ordinary mixing in a Turbula (type T2F, Willy A. Bachofen, AG Maschinenfabrik, Basel, Switzerland). The cylindrical mixing chamber is subjected to intensive, periodic movements, generated by rotation, translation and inversion according to the Schatz geometry theory (Figure 2). This movement is supposed to simulate the agitation achieved by manual shaking. The rotation speed can be set to 22,32 , 46, 68 and $96 \mathrm{rpm}$ [6]. In this study, the powders $(10 \mathrm{~g})$ were treated with a Turbula for $4 \mathrm{~min}$ (the time required to ensure homogenous mixing) at $46 \mathrm{rpm}$.

In addition, the active substance and magnesium stearate were also mixed by manual shaking in a bottle. In all, this provides three different processing methods to facilitate comparison.

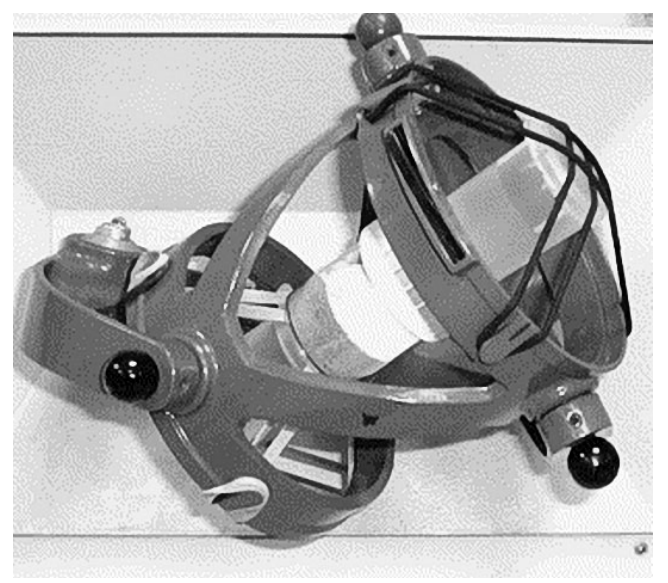

Fig. 2: The Turbula Mixer.

\subsection{Evaluation of Coating Material}

A standard Malvern Mastersizer fitted with a dry powder feeder has been used to evaluate the strength of 
the host-guest interaction. In this apparatus, powder de-agglomeration is controlled by adjusting the dispersion air pressure. The liberation of small guest particles from host particles can be achieved by increasing the air pressure, and the presence of liberated fine particles mixed with guest particles can be detected from differences in volume and number distributions. Five different dispersion air pressures were used: $0.5 ; 1,5 ; 2,5 ; 3.5$ bar and the maximum pressure of 4 bar. Complementary examination was undertaken using a Philips XL 30 ESEM.

\section{Results and Discussion}

\subsection{Surface Morphology of Coated Particles}

ESEM photos were recorded to examine the surface morphology of particles coated with $7 \%$ magnesium stearate using a Nara Hybridizer, Turbula and manual shaker (Figure 3).

It can be seen that each of the hybridizer, Turbula, and manual shaker facilitates the coating of the harpagophytum. However, in the case of manual shaking the particles of MgSt appear to be just lying on the harpagophytum surface whereas the particles resulting from hybridizer treatment have the $\mathrm{MgSt}$ firmly attached onto the surface of the host particles. This observation is supported by the number size distributions presented in Sec. 3.2.

\subsection{Strength of Interaction between Host and Guest Particles}

The method used to evaluate the strength of interaction between host and guest particles is based on the particle size analysis undertaken using a Malvern Mastersizer in dry feed mode. The liberation of guest particles is made possible by increasing the air pressure. Two different weight mass of magnesium stearate were used to undertake this study: 1 and $7 \%$.

\subsubsection{Harpagophytum $+7 \%$ of Magnesium Stearate}

Figure 4 shows the number size distributions for different dispersion air pressures, of harpagophytum with $7 \%$ (w/w) magnesium stearate, prepared by using the three different devices described above.

The number size distributions obtained at 0.5 bar dispersion air for the products coated using the different devices are similar. For 1.5 bar pressure, the particles resulting from the Turbula Mixer are displaced and par-
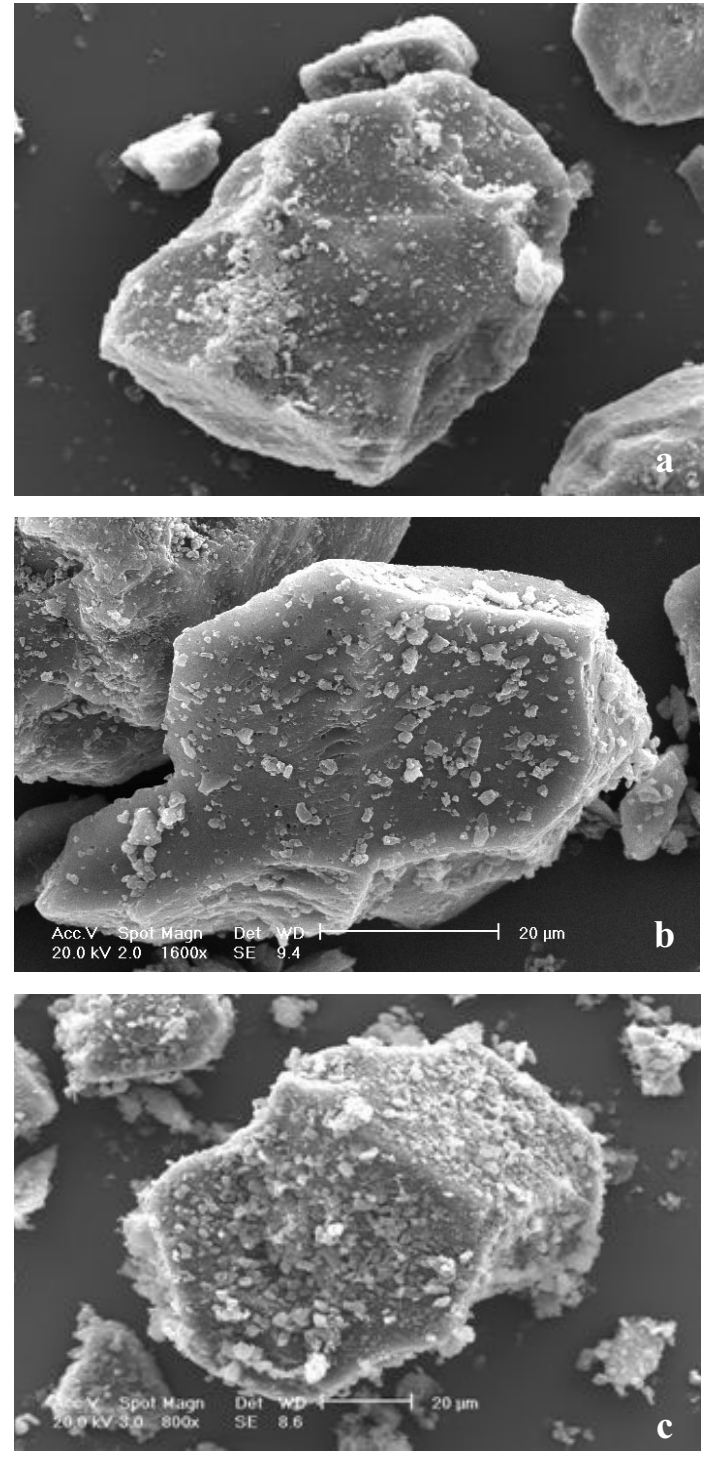

Fig. 3: Surface morphology of particles coated using the (a) Hybridizer, (b) Turbula and (c) manual shaker.

ticles are smaller than those obtained from the manual shaker and hybridizer. Therefore, at this pressure, the coating made by the Turbula Mixer, has weaker interactions between the host-host particles. For 2.5 bar pressure, the number size distributions for the particles coated by the Turbula Mixer have the same form as the MgSt distributions. This means that for this dispersion air pressure, there is liberation of small guest particles from host particles. The number size distribution of particles coated using the manual shaking device has the same shape as the number size distribution of the particles coated using the hybridizer. The number size distribution for pressures of 3.5 and 4 bar, are all similar, and consequently, only the number size distribution for 3.5 bar has been presented. At this pressure, all of the number size distributions of coated particles are super- 

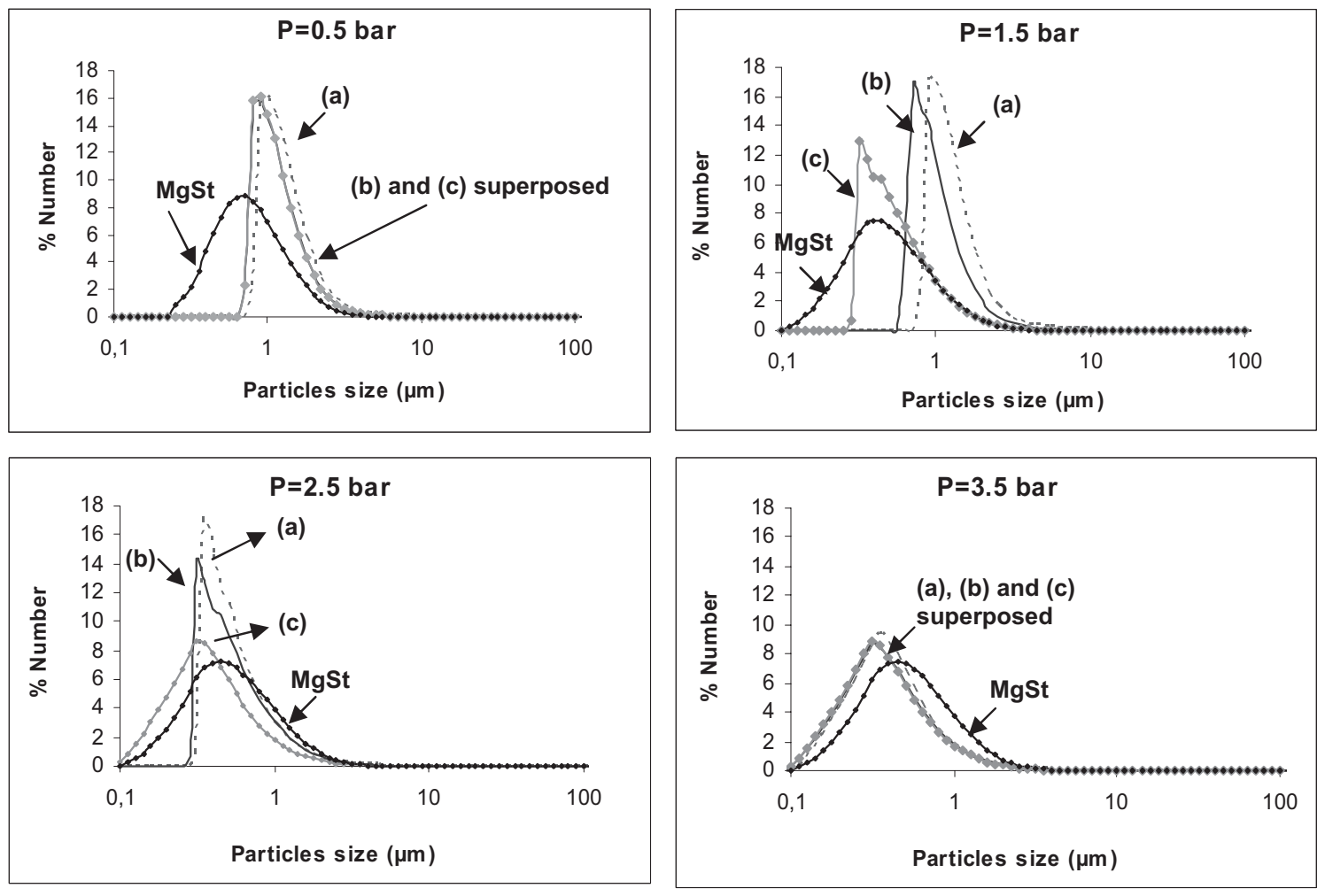

Fig. 4: Number size distributions of magnesium stearate $(\mathrm{MgSt})$ and particles coated with $7 \%$ magnesium stearate using: (a) Hybridizer, (b) manual shaker, and (c) Turbula.

imposed with the $\mathrm{MgSt}$ distribution, indicating complete liberation from the host particles.

The interactions between host and guest particles are found to be stronger when the hybridizer is used, because 3.5 bar pressure is required in order to deagglomerate the particles obtained, and the required deagglomerating pressure is 2.5 bar for particles coated using the Turbula Mixer.

The volume size distributions of particles coated with dispersion air pressures of 0.5 and $4 \mathrm{bar}$, are presented in Figure 5. The increase in dispersion air pressure results in an increased population of fine particles of size ca. $2 \mu \mathrm{m}$, and a decrease in the population with a size of ca. $50 \mu \mathrm{m}$. These results confirm the observations made with the number size distributions. The fine population is greater with the particles coated using the manual shaker and Turbula. This suggests that the quantity of guest particles obtained inside the collector in the hybridizer is not exactly $7 \%(\mathrm{w} / \mathrm{w})$. To explain this phenomenon, the electrostatic behavior of particles from the hybridi-
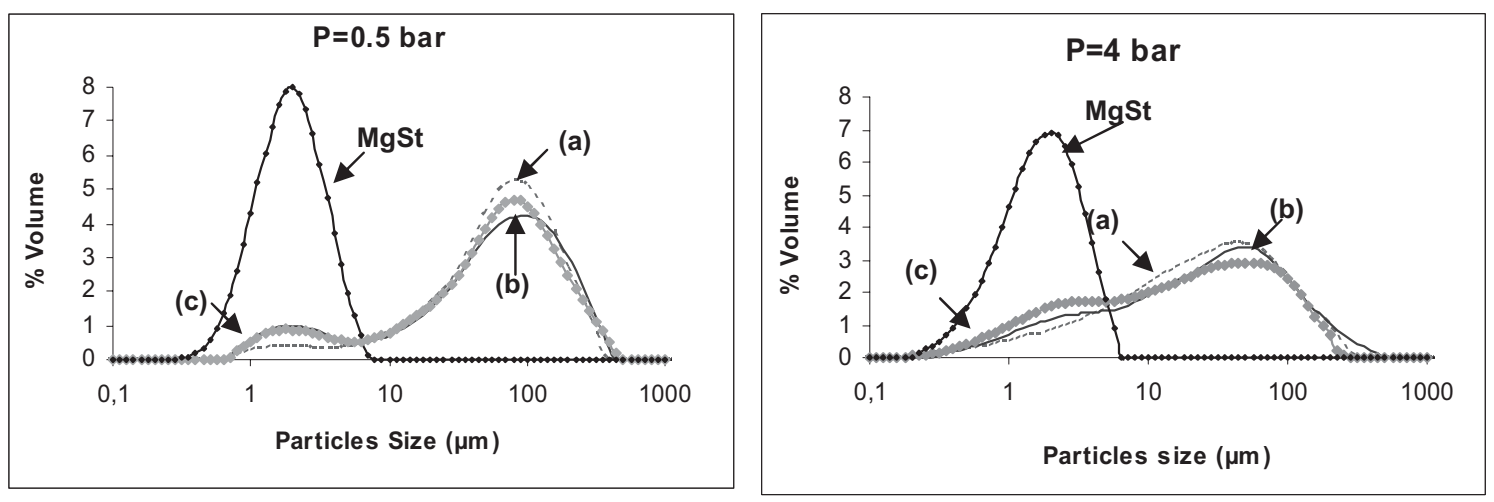

Fig. 5: Volume size distributions of magnesium stearate (MgSt) and particles coated with 7\% magnesium stearate using: (a) Hybridizer, (b) manual shaker, and (c) Turbula. 
zer was examined. It was observed that the harpagophytum and magnesium stearate were charged by friction with stainless steel.

\subsubsection{Harpagophytum $+1 \%$ of Magnesium Stearate}

The number size distribution for different dispersion air pressures, of harpagophytum with $1 \%(w / w)$ of magnesium stearate, prepared by using the different devices described earlier, is presented in Figure 6. For 0.5 bar, the particles treated in the Turbula are de-agglomerated because the number size distribution is similar to the number size distribution of magnesium stearate. The number size distributions of uncoated and coated particles produced using the Nara Hybridizer, are superimposed on pressures under 2.5 bar. At higher pressures, there is a displacement of particles coated using the Nara Hybridizer. The comparison of the surface of particles coated with $1 \%$ (Figure 7 ) and $7 \%$ (Figure 3 ) magnesium stearate, under the same operating conditions, suggest the existence of a film.

The volume size distribution of particles coated with $1 \%$ magnesium stearate is presented in Figure 8 . The

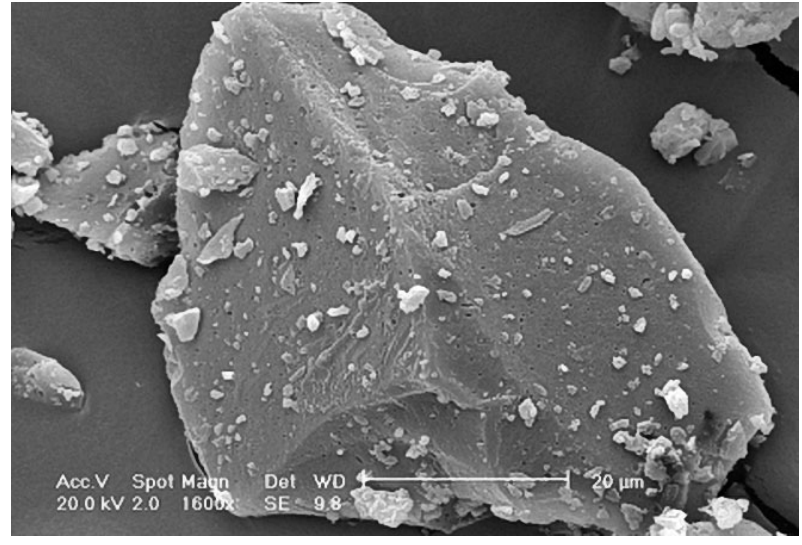

Fig. 7: Surface morphology of particles coated with $1 \%$ magnesium stearate using the Hybridizer.

population of particles with a size of ca. $2 \mu \mathrm{m}$ increases as the pressure increases.

In conclusion, the interactions between particles resulting from the hybridizer treatment are stronger than those resulting from other treatments because a greater pressure is required to de-agglomerate particles, as can be seen in Table 1.
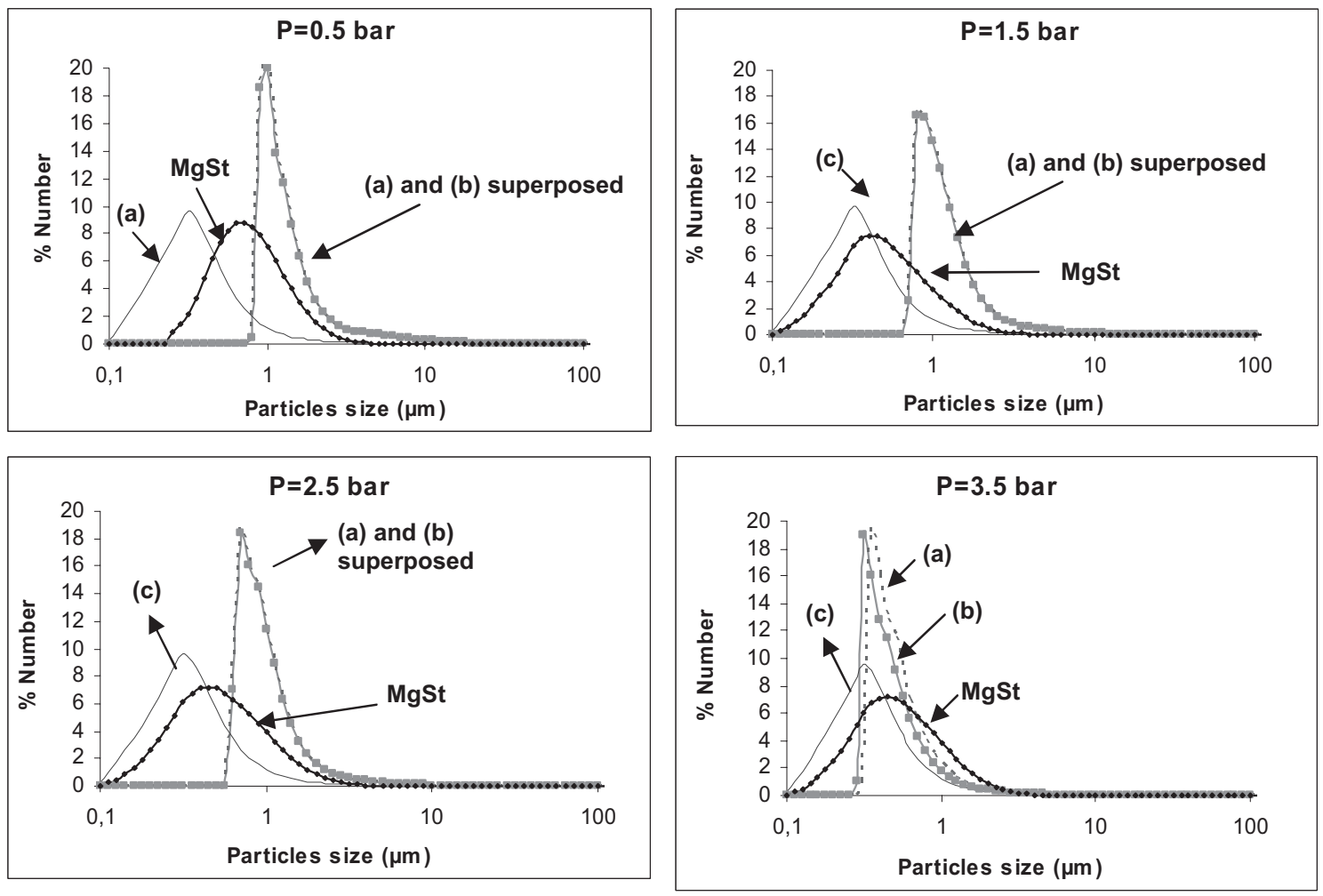

Fig. 6: Number size distributions of magnesium stearate (MgSt), uncoated particles (a) and particles coated with $1 \%$ magnesium stearate using: (b) Hybridizer, and (c) Turbula. 

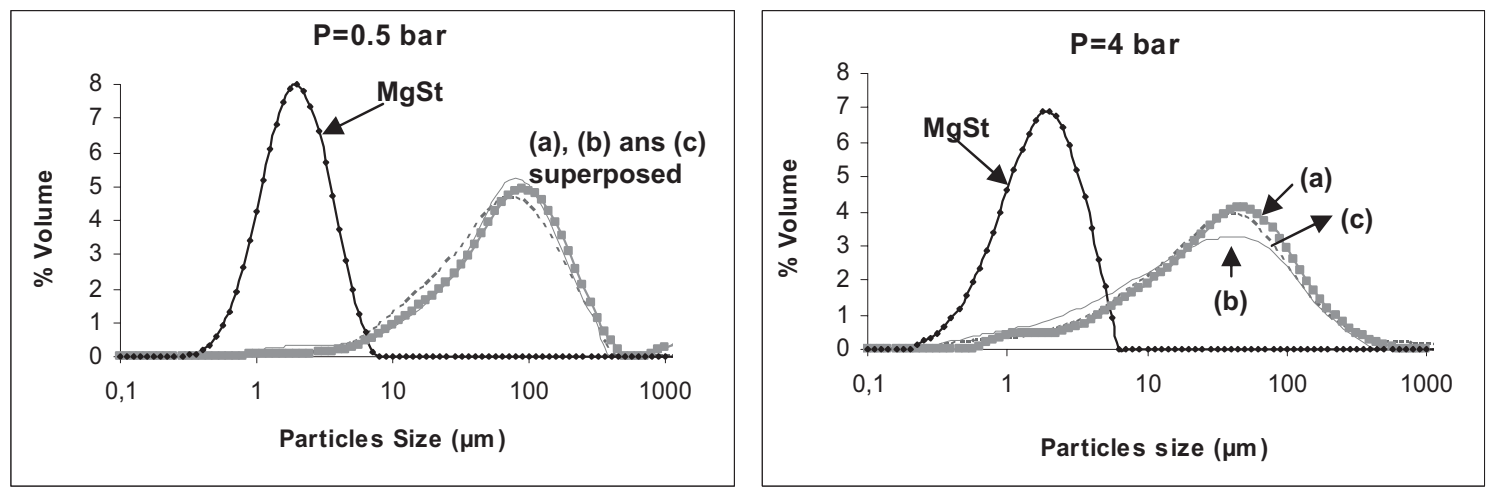

Fig. 8: Volume size distributions of magnesium stearate (MgSt), uncoated particles (a) and particles coated with $1 \%$ magnesium stearate using: (b) Hybridizer, and (c) Turbula.

Table 1: Dispersion air pressure required to de-agglomerate particles.

\begin{tabular}{|l|c|c|c|}
\hline Product & Hybridizer & Turbula & Manual shacking \\
\hline Harpagophytum $+1 \% \mathrm{MgSt}$ & $>4 \mathrm{bar}$ & $0.5 \mathrm{bar}$ & - \\
\hline Harpagophytum $+7 \% \mathrm{MgSt}$ & $3.5 \mathrm{bar}$ & $2.5 \mathrm{bar}$ & $2.5 \mathrm{bar}$ \\
\hline
\end{tabular}

\section{Conclusion}

The coating of a host powder by guest particles can be achieved by many different means ranging from the simple stirring together of the two components, to high energy impact coating in devices such as a Nara Hybridizer. The quality of the powder coating resulting from such methods is an important parameter, which depends on the physico-chemical interactions between the host and guest particles. To evaluate the strength of these interactions, a method based on particle size analysis using a standard Malvern Mastersizer with dry powder feed was developed. The surface modifications were observed using an environmental scanning electron microscope.

It is shown that effective coating can be undertaken by simple stirring and by using a Turbula Mixer and Nara Hybridizer. If the products obtained by simple stirring and from using a Nara Hybridizer are compared, it is found that the strength of the interaction between guest-host particles is stronger due to the mechanical forces generated by the Nara Hybridizer.

\section{Acknowledgements}

The authors wish to thank Christine Rolland, Severine Patry, Sylvie Del-confetto, Elisabeth Rodier and Laurent Devrient for their help and assistance. This work was supported by the Groupe Pierre Fabre.

\section{References}

[1] M. Ramlakhan, C. Wu, S. Watano, R. Dave, R. Pfeffer, Dry particle coating using magnetically assisted impaction coating: modification of surface properties and optimization of system and operating conditions, Powder Technol., 2000, 112, 137-148.

[2] A. Vilela, Powder, Influence d'une action mécanique en voie sèche sur l'amélioration et l'association d'actifs pharmaceutiques et d'excipients: application de l'Hybridizer Nara, PhD Thesis, Faculté de Pharmacie, Université Paris Sud XI et Faculté de Pharmacie de Châtenay-Malabry, 2005.

[3] R. Pfeffer, R. Dave, D. Wei, M. Ramlakhan, Synthesis of engineered particulates with tailored properties using dry particles coating, Powder Technol., 2001, 117, 40-67.

[4] M. Anderson, E. Santos, M. Seabra, A. Silva, S. Tufik, Evaluation of acute and chronic treatments with Harpagophytum procumbens on Freund's adjuvant-induced arthritis in rats, J. Ethnopharmacology, 2004, 91, 325330.

[5] S. Kangwantrakool, K. Shinohara, Preparation of new WC-Co/Ti-Al2O3 composite materials with mechanically coated particles, J. Chem. Eng. Japan, 2001, 34, 1486-1492.

[6] P. Porion, N. Sommier, A. Faugere, P. Evesque, Dynamic of size segregation and mixing of granular materials in 3D-blender by NMR imaging investigation, Powder Technol., 2004, 141, 55-68.

[7] W. Pieper, The Nara hybridization System: Design your own particle, News from industry, 1996, 8, 232-234. 